\title{
Senderos Pedagógicos
}

\section{Intuiciones acerca del infinito en docentes y estudiantes de 15 a 17 años ${ }^{1}$}

\author{
Intuitions about Infinity in Teachers and Students Aged 15 to 17
}

Autor: Jeferson Alexander Orozco ${ }^{2}$

Recibido: $18 / 04 / 2018$

Aprobado: 31/03/2019

1. Resultado de investigación Universidad Pedagógica y Tecnológica de Colombia.

2. Aspirante a Magister en Educación Matemática Universidad Pedagógica y Tecnológica de Colombia. jeferson.orozco.pinzon@gmail.com

\section{Resumen}

Los docentes en las actividades cotidianas, entre las que se encentran las relacionadas con las temáticas de clase, tratamos con los estudiantes temas que tienen inmerso el concepto infinito, o por lo menos lo hemos mencionado, pero realmente sabemos ¿Qué piensa un docente o un estudiante de grado $11^{\circ}$ acerca del infinito? o ¿Qué piensan del concepto de límite? En este trabajo se presentan las concepciones que sobre el infinito exponen los estudiantes con edades de 15 a 17 años (Grado $11^{\circ}$ ), así como, las de los docentes de matemáticas. Para tal fin, se identifican y categorizan las concepciones del concepto de infinito a partir de dos cuestionarios.

Palabras clave: ilimitado, indefinido, infinito, infinito potencial y actual, concepciones. 


\begin{abstract}
As teachers we are constantly teaching topics that have to do with the concept of -infinite- or at least we have talked about it in our lessons, however, I wonder if we really know zwhat an eleventh grader or a mathematics teacher thinks about the definition of infinite? or ¿what are their notions about the concept of limit? Due to this, in this research we share the
\end{abstract}

conceptions that mathematics teachers and eleventh graders (15 to 17 years old) have about the concept of infinite. For this purpose, the definitions of infinite are identified and categorized from two surveys.

Keywords: Unlimited, indefinite, infinite, infinite potential and actual, conceptions.

\section{Introducción}

La noción de infinito tiene orígenes eminentemente filosóficos, el término en sí, lejos de pretender cuantificar, aspiraba a calificar cualquier ente inaccesible o proceso indefinido. Aún ocurre así en determinados contextos. Pero las diversas épocas racionalistas de la historia del pensamiento han intentado definir de manera estricta el alcance de dicho concepto (Martínez, 2009).

Dado que el concepto de infinito ha estado inmerso en el trabajo de célebres matemáticos a lo largo de la historia, se ha reflexionado en él desde épocas muy tempranas en la antigüedad griega y, es desde la matemática donde se ha logrado una mejor comprensión de esta temática; entonces para una mejor lectura y asimilación de este objeto matemático es necesario hacer un recorrido histórico sobre este concepto, ya que ha tenido un lugar determinante en el desarrollo conceptual de las matemáticas.

Distintos investigadores han trabajado en reconocer los obstáculos y dificultades que posee la enseñanza y aprendizaje del infinito, porque este es necesario en la comprensión del cálculo infinitesimal. Son varios los factores que influyen en la concepción de infinito, estos se describen más detalladamente en el planteamiento del problema. Un factor que predomina es el de las intuiciones que tiene cada sujeto, las cuales son construidas y apoyadas en la experiencia y el conocimiento, además, son coherentes con el infinito potencial y se convierten en dificultades para entender objetos matemáticos que requieren de un infinito en acto.

En este artículo se presenta el desarrollo y resultados de un estudio realizado sobre las concepciones acerca del infinito matemático de estudiantes de 15 a 17 años y de docentes del área de matemáticas. Contó con la participación de 60 
estudiantes y 7 docentes del Colegio Cooperativo Reyes Patria de la ciudad de Sogamoso Boyacá, entre los meses de septiembre y octubre de 2017.

En la primera parte se presenta el marco teórico que sustenta nuestra investigación, luego aparece la metodología utilizada, después una descripción y categorización de las concepciones de los estudiantes respecto al infinito, luego un análisis y categorización para las concepciones de los docentes que evidencian algunos protocolos y, por último, se presentan algunas conclusiones generales.

\section{Marco teórico}

El estudio de las concepciones del infinito matemático se sustenta en las teorías sobre concepto de Vergnaud (1990b, p.9, citado por D'Amore, 2006), infinito actual e infinito potencial (Dubinsky, Weller, McDonald y Brown, 2005a, p. 346), Ilimitado-Indefinido-Infinito (Arrigo, D'Amore y Sbaragli, 2011, p.15), dificultades y obstáculos (Arrigo y D’Amore, 1999).

Vergnaud (1990b) parte de la definición de concepto matemático: un concepto es una terna de conjuntos (S, I, S) en donde S: es el conjunto de situaciones que dan sentido al concepto (el referente), I: el conjunto de los invariantes en los que se basa la operatividad de los esquemas (el significado) y, S: el conjunto de representaciones simbólicas usadas para presentar el concepto, sus propiedades y las situaciones a las que se refiere. Igualmente, una concepción estaría formada por esta misma terna, pero considerándola en un momento dado de la evolución del concepto (Vergnaud, 1990b, p. 50).

Para definir obstáculo, Arrigo y D’Amore (1999) afirman que:

(...) obstáculo no es en sí mismo asimilable a un error; el obstáculo es una idea que, en el momento de la formación de un concepto, fue eficaz para afrontar problemas precedentes (incluso solo cognitivos), pero que se revela ineficaz cuando se intenta aplicarla a una nueva situación (p. 4).

En este sentido, estos dos autores declaran los obstáculos y dificultades más importantes que se han observado alrededor de la enseñanza y aprendizaje del concepto de infinito:

- Obstáculos epistemológicos:

- Dependencia: convicciones intuitivas que llevan a los estudiantes a pensar que en un segmento largo existan más puntos que en uno corto (Arrigo y D’Amore, 1999, p.4). 
- Aplastamiento: todos los conjuntos infinitos tienen la misma cardinalidad (Arrigo y D’Amore, 1999, p.4).

- Aceptaciones intuitivas de dependencia y aplastamiento se hallan en contradicción entre ellas: la dificultad que encuentra el estudiante para darse cuenta cuando dos afirmaciones están en contradicción y, aún más, la casi total indiferencia que demuestra si se da cuenta de dicha contradicción (Arrigo y D'Amore, 1999, p.5).

- Concepción del infinito potencial vs. Concepción del infinito actual: este debate ha inspirado diferentes investigaciones, por ejemplo, las de Moreno y Waldegg (1991), Shama y Movshovitz Badar (1994). Se han hallado, en verdad, resultados a veces contradictorios; pero está probado que la evolución de la concepción actual del infinito matemático es más lenta, y se da en modo contradictorio a lo largo del curso del currículo escolar, gracias a un proceso de maduración y sistematización cognitiva de los aprendizajes. Este tipo de dificultad no se encuentra solo entre estudiantes, sino también entre profesores (en formación), lo que refuerza la necesidad de tomar siempre más en examen los obstáculos didácticos y los contenidos disciplinares de la formación (D’Amore, B. 2013, p. 26).

- Obstáculos didácticos:

- Ausencia en el currículum: la enseñanza del infinito no aparece como tal en el currículum de educación básica ni de educación media (Arrigo y D’Amore, 1999, p.4).

- Formación del docente: la concepción que muchos docentes tienen acerca del infinito es la noción intuitiva, debido a la formación del concepto que han desarrollado durante su aprendizaje (Arrigo y D’Amore, 1999, p.4).

- Discurso docente: derivado de los dos puntos anteriores, algunos docentes le huyen a la construcción del concepto con los estudiantes, lo que lleva a dar por entendido en muchas ocasiones esta noción con toda la problemática que ello conlleva (Arrigo y D’Amore, 1999, p.4).

- Deslizamiento: no se acepta una demostración en la cual se pasa de un objeto de discurso a otro; por ejemplo, se habla de hechos geométricos y se pasa a consideraciones aritméticas. Otra pauta sería, la dificultad de aceptar la correspondencia biunívoca entre el conjunto de los números naturales (N) y el subconjunto de los números cuadrados (Arrigo y D’Amore, 1999, p.4).

\section{Infinito potencial e infinito actual}

En cuanto a infinito potencial e infinito actual, se han hecho bastantes investigaciones dentro de la didáctica de la matemática. Estas son algunas posturas: 
El infinito potencial es la concepción de infinito como un proceso (Dubinsky et al., 2005a, p. 346). Este proceso se construye empezando por los primeros pasos (por ejemplo 1, 2, 3 en la construcción del conjunto de los números naturales) que se refieren a una concepción acción. Repetir estos pasos (por la adición de 1 repetidamente) al infinito, requiere de la interiorización de estas acciones en un proceso. El infinito actual es el objeto mental que se obtiene de la encapsulación de este proceso (Dubinsky et al., 2005a, p. 346).

Una posición coherente con las anteriores definiciones de infinito actual y potencial es la siguiente: "actual" significa que el infinito está presente en un acto único, todo a la vez, como un dato de hecho; mientras que "en potencia”, significa que a pesar de que una situación aparezca como finita en el instante en el cual se refiere, existe la seguridad de que se puede ir más allá del límite establecido (que, por lo tanto, no es definitivo): "Una cosa viene de otra sin fin, y cada una de ellas es finita, pero siempre hay nuevas” (Arrigo, D’Amore y Sbaragli, 2011, p.28).

De otro lado, ilimitado, indefinido, infinito son palabras que tienen actualmente distintos significados (Arrigo, D’Amore y Sbaragli, 2011, p. 15):

Ilimitado: algo que no tiene límite, como se asume la recta entendida en su extensión lineal.

Infinito: se puede entender como una cantidad numérica mayor que cualquier cantidad natural.

Indefinido: se refiere a algo sin confín claro, o bien sin una definición precisa.

\section{Metodología}

La investigación fue de tipo cualitativo y con un enfoque fenomenológico hermenéutico (Fiorentini y Lorenzato, 2010), que se fundamenta filosóficamente en la fenomenología y en el proceso hermenéutico de la interpretación. Además, parte de la suposición de que la solución de problemas educativos pasa inicialmente por la búsqueda de interpretación y comprensión de los significados atribuidos por los implicados (los sujetos que viven el fenómeno). Eso puede suceder por medio de un proceso de investigación que consiste en revelar mecanismos y significados ocultos, alcanzando, así, la esencia de los fenómenos” (p. 39).

Este trabajo se desarrolló en el Colegio Cooperativo Reyes Patria de la ciudad de Sogamoso durante el mes de septiembre de 2017, y la temática que estaban trabajando los estudiantes eran derivadas de funciones trigonométricas; por lo tanto, ya se había tratado el tema de límites y continuidad. Participaron siete docentes pertenecientes al área de matemáticas de la institución; dichos docentes 
son licenciados en matemáticas, licenciados en matemáticas y estadística, y áreas afines (tres docentes de primaria y cuatro de bachillerato). También participaron todos los estudiantes matriculados (60) en grado undécimo para el año 2017, y cuyas edades varían entre los 15 y 17 años, sus niveles socioeconómicos se clasifican en medio y alto. La institución es de carácter privado.

Dentro del estudio de las concepciones del infinito se plantean tres etapas que validarán el objetivo planteado.

Etapa 1: intuición acerca del infinito por parte de los estudiantes. Lo que se quiere en esta primera parte es conocer lo que intuyen los estudiantes en cuanto al infinito. Para ello, se aplica un cuestionario (ver anexo A) con cuatro preguntas abiertas donde notaremos en que contextos asocian el concepto para luego categorizar las intuiciones, y conocer las definiciones que se dan a infinito, ilimitado e indefinido.

Etapa 2: identificación del infinito en matemáticas por parte de los estudiantes. Luego de conocer algunas intuiciones de los estudiantes, se desarrolla un segundo cuestionario (véase anexo B) con cuatro preguntas; tiene como objetivo ver el infinito en un contexto matemático y determinar si el estudiante puede establecer una correspondencia biunívoca en situaciones que tienen implícito el infinito.

Etapa 3: intuición acerca del infinito por parte de los docentes. Se aplica un cuestionario (ver anexo A) a siete docentes del área de matemáticas (D1, D2, D3, D4, D5, D6, D7) de la misma institución educativa, describiendo y categorizando sus intuiciones.

\section{Resultados}

Se presenta un análisis de las respuestas por parte de los estudiantes, a cada una de las preguntas de los cuestionarios de los anexos A y B. También se presenta un análisis de las respuestas por parte de los docentes, a cada una de las preguntas del cuestionario del anexo A.

\section{Etapa 1: intuición acerca del infinito en estudiantes}

Luego de aplicado el primer cuestionario (véase anexo A), analizamos las respuestas más reiteradas en cada una de las cuatro preguntas que conforman dicho cuestionario, como se muestra a continuación.

Pregunta 1. Desde tu percepción podrías decirme: ¿Qué es el infinito? y ¿Por qué es infinito? Las siguientes son las afirmaciones más comunes dentro de los 
estudiantes, respecto a lo que consideran sobre el infinito: no tiene un final, no tiene un principio ni final, algo que no es finito o no se puede alcanzar, es desconocido y sin fin, es algo que tiene inicio, pero no final, nunca se acaba. En las respuestas de los estudiantes también se puede notar que confunden el infinito con ilimitado o, el infinito, con indefinido. En la figura 1 se pueden observar algunas de estas respuestas de los estudiantes.

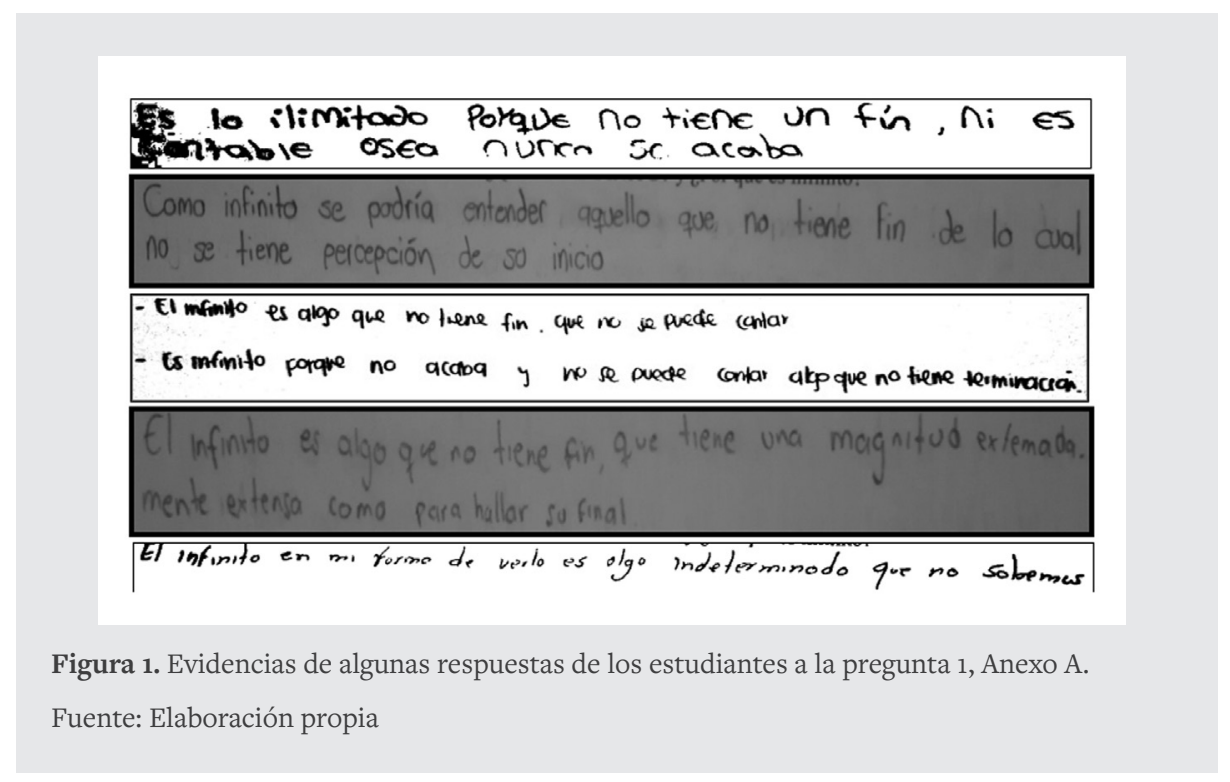

Además de las confusiones entre infinito, ilimitado e indefinido, se nota que los estudiantes atribuyen otra naturaleza a este concepto, puesto que se les dificulta explicarlo; en todas las afirmaciones se ve un claro proceso que nunca termina (infinito potencial), y al tratar de argumentar el ¿por qué es infinito? vuelven a contestar que es un proceso sin fin, sin límite, sin terminación (Infinito potencial).

Solo cuatro estudiantes, ante esta primera pregunta, contestaron en un ámbito matemático, pero también se encuentra claramente implícito el concepto de infinito en potencia, como se puede observar en la figura 2. 


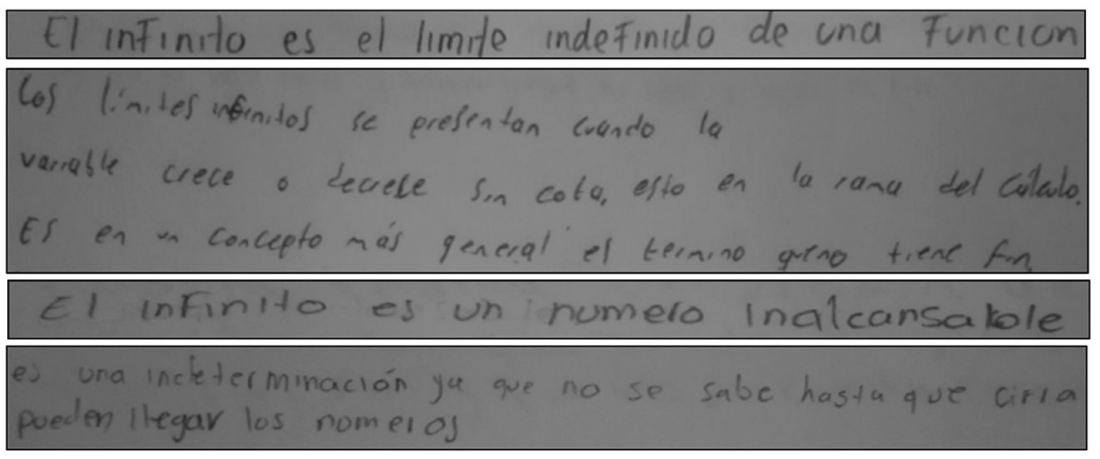

Figura 2. Evidencias de algunas respuestas de los estudiantes a la pregunta 1, Anexo A. Fuente: Elaboración propia

Pregunta 2. ¿Cómo explicarías a una persona el concepto de infinito? Algunos estudiantes responden algo muy similar a la respuesta sobre la pregunta 1, siendo coherentes con su postura potencial hacia el infinito, lo que deja ver la dificultad al argumentar respecto a este concepto. Algunas respuestas a esta pregunta se pueden ver en la figura 3 .

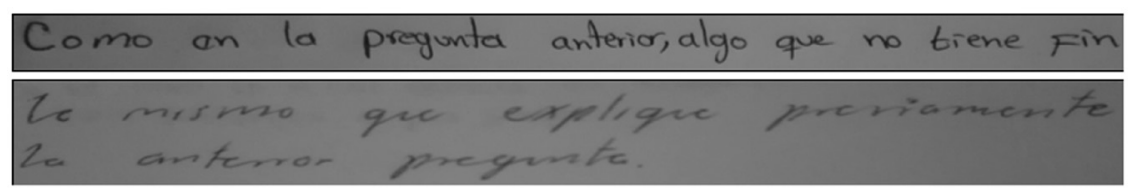

Figura 3. Evidencias de algunas respuestas de los estudiantes a la pregunta 2, anexo A. Fuente: Elaboración propia

Por otra parte, varios estudiantes muestran distintos contextos que utilizarían a la hora de explicar el infinito, como se puede evidenciar en la figura 4. 

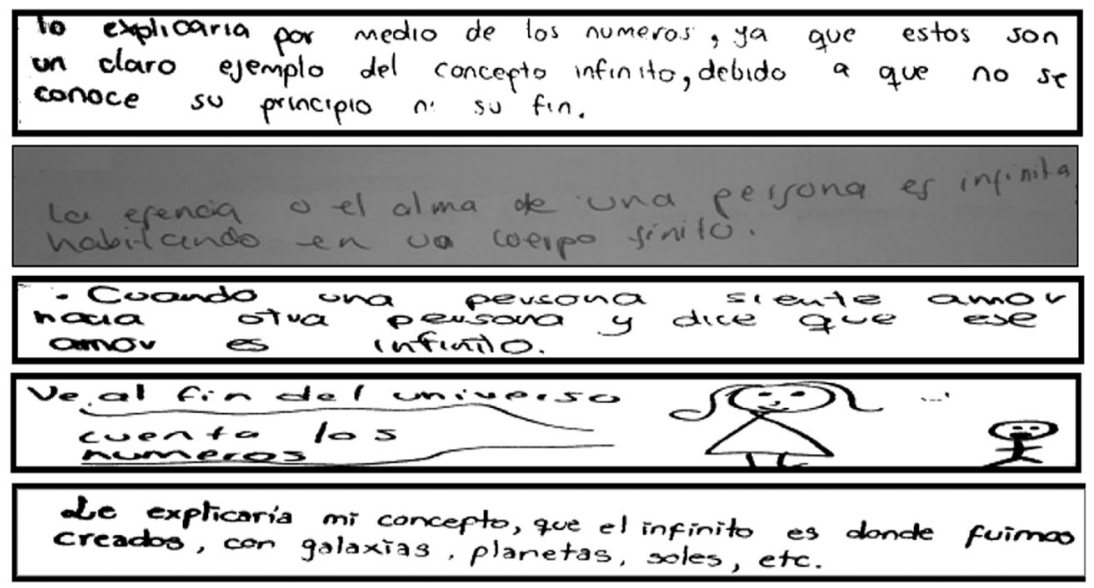

Figura 4. Evidencias de algunas respuestas de los estudiantes a la pregunta 2, Anexo A. Fuente: Elaboración propia

Las anteriores intuiciones están muy lejos del concepto de infinito, también dificultarían el aprendizaje del mismo; además, reflejan distintos obstáculos didácticos como el discurso docente o la formación docente, pues, se ha permitido que cada estudiante cree una concepción errónea, al no reflexionar sobre este tema durante sus anteriores años de escolaridad.

Pregunta 3: Escribe cinco frases que contengan la palabra infinito. A continuación, categorizamos las respuestas que han dado los estudiantes a estas preguntas y a las preguntas 1 y 2, que corresponden a la intuición que se tiene acerca del infinito. Citamos las más significativas y reiteradas.

Categoría A: Los sentimientos, el amor, el odio, .... Amo a mi mamá hasta el infinito, te quiero hasta el infinito, mi odio hacia Uribe es infinito, mi amor por ella es infinito, el amor por el aprendizaje debería ser infinito, el saber y el sentir son infinitos, la imaginación es infinita.

Categoría B: Las matemáticas, límites, los números, .... Los números son infinitos, el límite de una función es infinito, un número sobre cero es infinito, límite cuando x tiende al infinito, el infinito se representa ( $\infty)$, e1 dividido en o es infinito, infinito son todos los valores que se tienen en $[7, \infty)$ a veces la clase de matemáticas parece infinita, las soluciones del sistema de ecuaciones son infinitas. 
Categoría C: La mística, el alma, la eternidad, .... La paciencia de Dios es infinita, el ser humano no es infinito, el Espíritu Santo es infinito, la vida es infinita, el alma es infinita.

Categoría D: El universo, los planetas, las galaxias, .... El universo es infinito, el espacio exterior es infinito, el infinito tiene galaxias, existen infinitas estrellas.

Categoría E: Las ciencias naturales, .... Los átomos son infinitos, masa infinita, fuerza infinita, las cargas positivas y negativas son infinitas, los granos de arena son infinitos, el circuito es infinito, el tiempo es infinito, las preguntas sobre nuestro origen son infinitas. Otra frase de las más usadas en este punto es "Hasta el infinito y más allá”, con referencia a Buzz un personaje cinematográfico de la saga Toy Story.

Pregunta 4. ¿Son iguales o diferentes estas tres palabras: indefinido, ilimitado, infinito? 36 de los 60 estudiantes responden que son palabras iguales y utilizan frases como: son sinónimos, son similares, se complementan, expresan lo mismo... Además de considerar iguales las palabras, presentan una postura mística ante las mismas y utilizan el concepto de indefinido e ilimitado para explicar el infinito (véase figura 5).

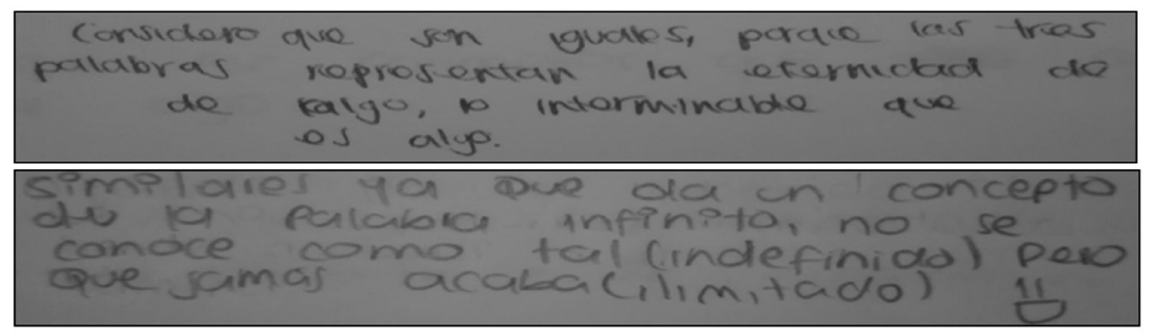

Figura 5. Evidencias de algunas respuestas de los estudiantes a la pregunta 4, Anexo A. Fuente: Elaboración propia

Recordemos que indefinido, ilimitado e infinito, según diversas investigaciones, se consideraban sinónimos en tiempos de Anaximandro de Mileto (610 a. C. - 547 a. C.) y, actualmente, tienen distintos significados. Por lo tanto, son demasiados los estudiantes que consideran iguales estas tres palabras. Algunos estudiantes responden que son palabras distintas, pero al argumentar el porqué, se contradicen aclarando que infinito es igual a ilimitado o infinito es indefinido (véase figura 6). 


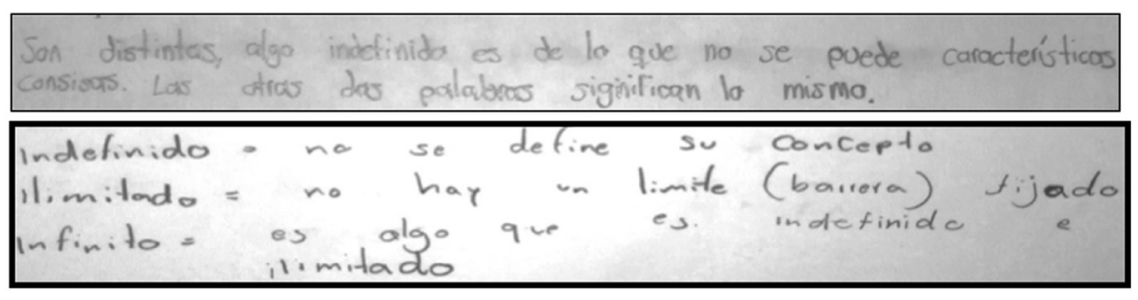

Figura 6. Evidencias de algunas respuestas de los estudiantes a la pregunta 4, Anexo A. Fuente: Elaboración propia

Algunos estudiantes responden que son palabras distintas, pero al argumentar el porqué, se contradicen cuando aclaran que infinito es igual a ilimitado o infinito es indefinido (véase Figura 7).

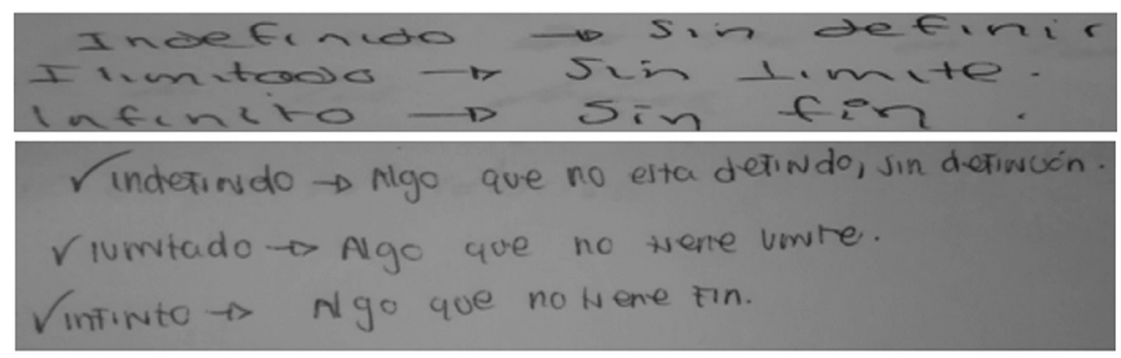

Figura 7. Respuestas de estudiantes a la pregunta 4, Anexo A.

Fuente: Elaboración propia

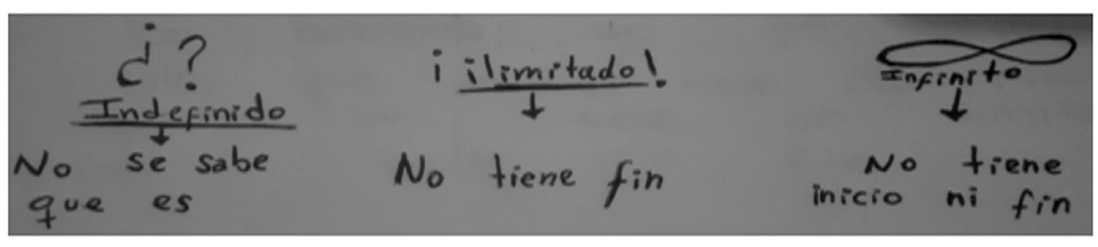

Figura 8. Respuestas de un estudiante a la pregunta 4, Anexo A.

Fuente: Elaboración propia 
Las palabras indefinido, ilimitado e infinito, según diversas investigaciones, se consideraban sinónimos en tiempos de Anaximandro de Mileto (610 a. C. - 547 a. C.) y actualmente tienen distintos significados como se indicaron en el marco teórico; entonces son demasiados los estudiantes que contemplan como iguales estas tres palabras, si se considera que han transcurrido más de dos mil años, lo que evidencia la poca discusión que esta temática ha tenido en las aulas de clase.

\section{Etapa 2: identificación del infinito en matemáticas}

Dentro de las cuatro preguntas correspondientes al segundo cuestionario (véase Anexo B), se encuentra que ninguno de los 60 estudiantes puede establecer una correspondencia biunívoca entre segmentos de distintas longitudes, entre segmento y semirrecta, entre segmento y recta o entre circunferencias de distintos radios. Se presentan las respuestas que más se repiten y las más significativas e interesantes, ya que, arrojan bastante información para entender la percepción que tienen los estudiantes en cuánto al infinito matemático.

Pregunta 1. La respuesta más usual es considerar el segmento CD con mayor cantidad de puntos al tener mayor longitud, como se puede ver en la figura 9. Otros estudiantes respondieron que en los dos segmentos hay la misma cantidad de puntos, pero haciendo referencia a los puntos de los extremos de los segmentos (véase figura 10).

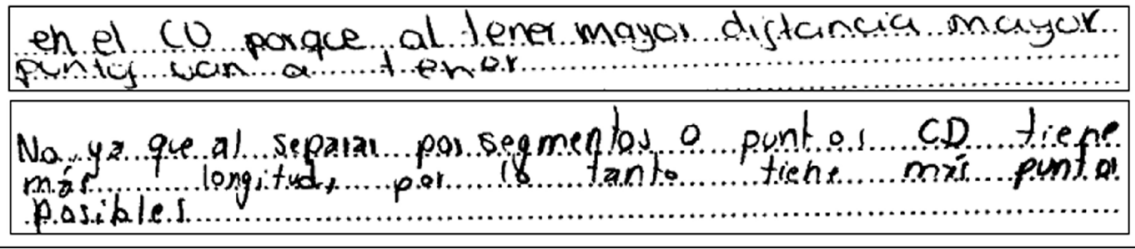

Figura 9. Evidencia de las respuestas de algunos estudiantes a la pregunta 1, anexo B Fuente: Elaboración propia 


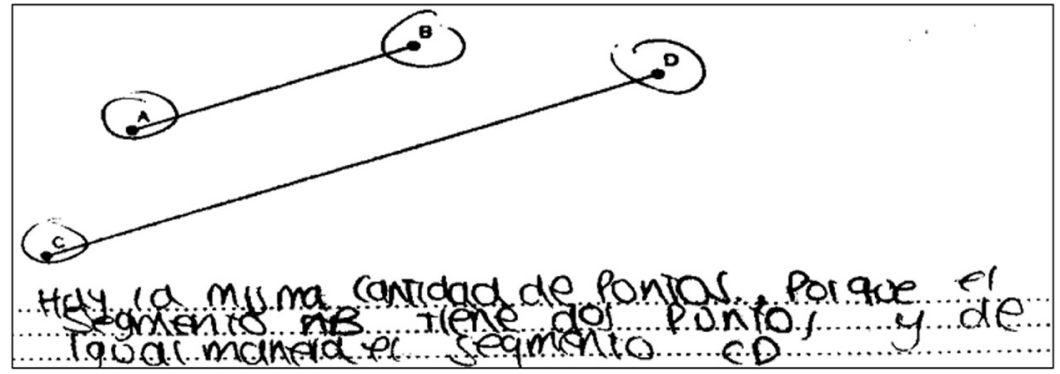

Figura 10. Evidencia de las respuestas de algunos estudiantes a la pregunta 1, anexo B. Fuente: Elaboración propia

En la Figura 9 se ve un claro ejemplo del obstáculo epistemológico llamado dependencia, obstáculo presente en la mayoría de los estudiantes, al considerar que en un segmento de mayor longitud existen más puntos que en uno de menor longitud. En la Figura 10 se evidencian obstáculos de tipo didáctico como la formación docente y el discurso docente, al ser varios los estudiantes que responden de esta misma manera.

Pregunta 2. La respuesta más común es considerar la semirrecta con mayor cantidad de puntos respecto al segmento, claro ejemplo de dependencia (véase figura 11).

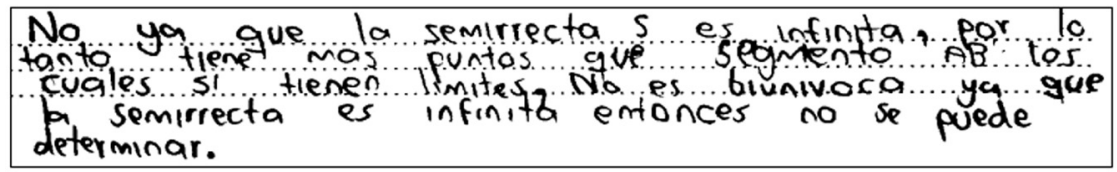

Figura 11. Algunas respuestas de los estudiantes, a la pregunta 2, anexo B. Fuente: Elaboración propia

Al igual que en la pregunta 1 de este segundo cuestionario, los estudiantes omiten la continuidad de puntos y solo tienen en cuenta los que delimitan; se observan obstáculos de tipo didáctico como formación docente y discurso docente (véase Figura 12). 


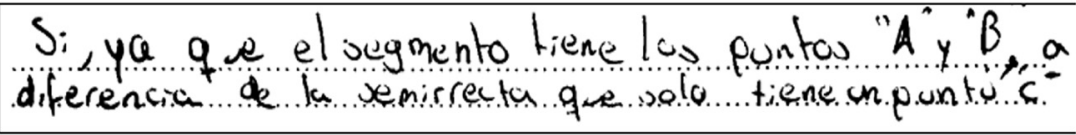

Figura 12. Algunas respuestas de los estudiantes, a la pregunta 2, anexo B.

Fuente: Elaboración propia

Pregunta 3. ¿Consideras que hay más puntos en el segmento $\mathrm{AB}$ que en la recta $r$ ?

Se resalta que un alto número de estudiantes considera que hay más puntos en la recta que en el segmento, es decir, obstáculo epistemológico dependencia, algunas de estas respuestas se pueden ver en la Figura 13. Otros estudiantes tuvieron en cuenta solo los puntos que delimitan el segmento, así, hay más puntos en el segmento que en la recta, o aún, llegaron a concluir que la recta no tiene puntos (véase Figura 14). Algo que hace probable que el docente en algún momento creo un obstáculo de tipo didáctico (discurso docente).

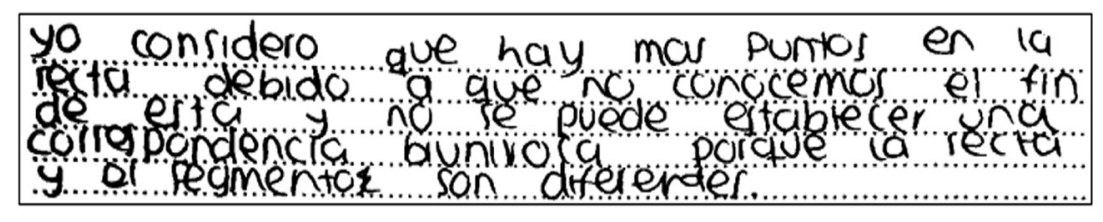

Figura 13. Algunas respuestas de los estudiantes, a la pregunta 3, anexo B. Fuente: Elaboración propia

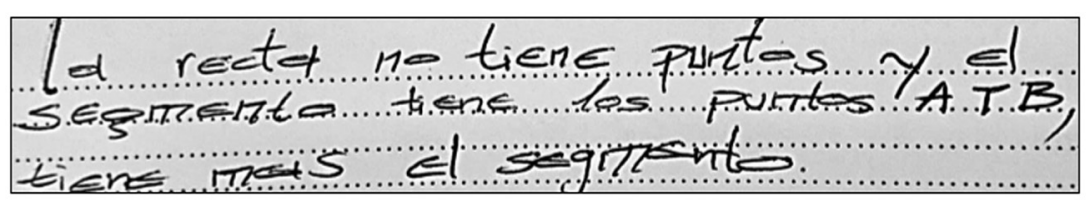

Figura 14. Algunas respuestas de los estudiantes, a la pregunta 3, anexo B.

Fuente: Elaboración propia 
Pregunta 4. ¿Consideras que hay más puntos en la circunferencia de radio $R$ o en la circunferencia de radio $s$ ?

$\mathrm{Al}$ igual que en las respuestas a las tres preguntas anteriores, la respuesta más usual a la pregunta 4 es afirmar que la circunferencia de radio mayor tiene más puntos (dependencia); varios estudiantes afirman que tiene igual cantidad de puntos, pues solo tienen en cuenta los puntos que se resaltan, y llegan a considerar el centro como parte de la circunferencia (véase Figura 15). Se evidencian también confusiones con respecto a longitud de circunferencia y área del círculo (véase Figura 16).

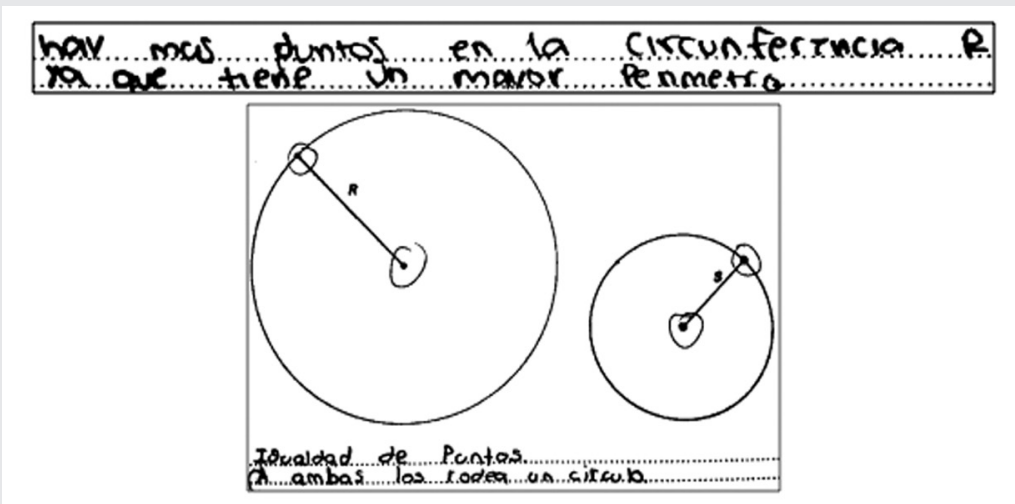

Figura 15. Algunas respuestas de los estudiantes, a la pregunta 4, anexo B. Fuente: Elaboración propia

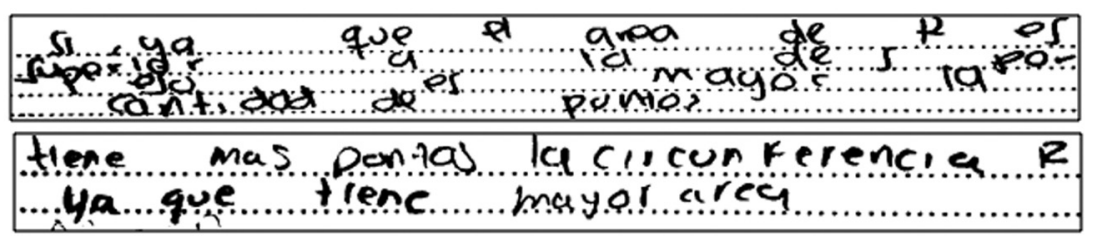

Figura 16. Evidencia de algunas respuestas de los estudiantes, a la pregunta 4, anexo B. Fuente: Elaboración propia 
Se observó en la Etapa 2, la influencia de algún docente durante la formación de este grupo de estudiantes en el área de matemáticas, al notar varias veces la similitud en sus respuestas al tener en cuenta solo los puntos resaltados en cada una de las situaciones ilustradas y omitiendo la continuidad de puntos.

\section{Etapa 3: intuición acerca del infinito por parte de los docentes}

Se aplicó el cuestionario (véase anexo A) a los docentes y se analizaron las respuestas significativas en cada una de las cuatro preguntas que conforman dicho cuestionario, como se muestra a continuación.

Pregunta 1: Desde tu percepción podrías decirme: ¿Qué es el infinito? y ¿Por qué es infinito?

Las siguientes son las concepciones que tienen los siete docentes (D1, D2, D3, ..., D7), las cuales transcribiremos textualmente:

D1: Es una situación inalcanzable y solo podría tener un concepto cercano (noción) en nuestra memoria o pensamiento.

D2: Qué es muy numeroso o muy grande sin límite.

D3: Algo que no tiene fin...

D4: No tiene fin, no tiene límite. Es infinito porque no posee la misma propiedad que un conjunto finito.

D5: Es la representación de la prolongación del conjunto de números, porque el infinito no tiene fin, siempre hay un número que lo prosigue.

D6: Un valor muy lejano desconocido. No tiene final, ni límite.

D7: Podríamos verlo desde la noción de cantidad, muy grande, imposible de contar.

En todas las definiciones proporcionadas por los docentes se ve un claro proceso que nunca termina (infinito potencial), algo muy similar a lo expuesto por los estudiantes (véase figura 17).

Pregunta 2: ¿Cómo explicarías a una persona el concepto de infinito?

Los docentes explicarían dicho concepto haciendo referencia a los contextos en los cuales utilizarían el concepto de infinito. Algunos protocolos se pueden observar en la figura 17. 


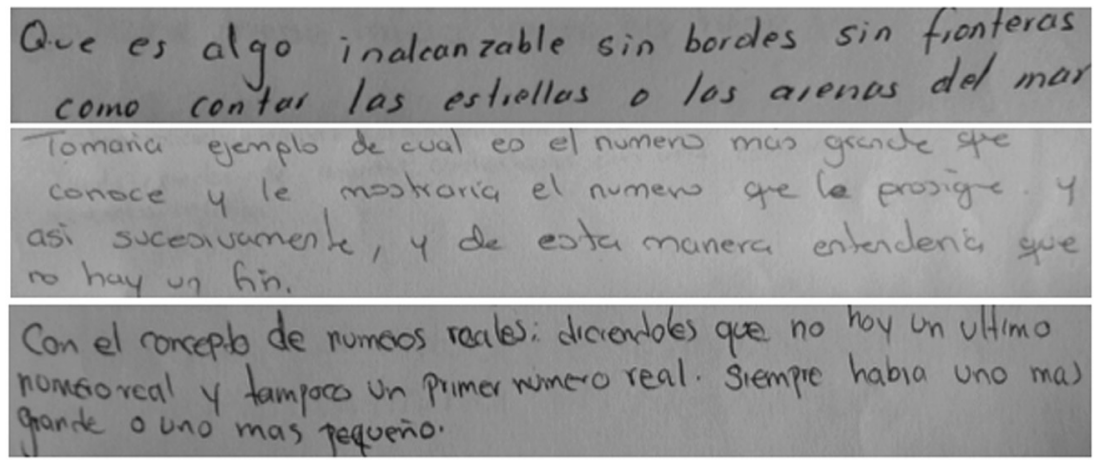

Figura 17. Evidencia de algunas respuestas de los docentes.

Fuente: Elaboración propia

Pregunta 3. Escribe cinco frases que contengan la palabra infinito. Categorizamos las afirmaciones expuestas por los docentes haciendo uso de la misma clasificación hecha para analizar la postura de los estudiantes. Esto debido a que son muy parecidas.

Categoría A: los sentimientos, el amor, el odio, .... El amor hacia un hijo es infinito, toda gran pasión termina en lo infinito, para ti un amor infinito o nada, me he perdido en la infinidad de tus ojos.

Categoría B: Las matemáticas, límites, los números, ... Los números son infinitos, los números naturales son infinitos, el número cero es un número infinito, hay números decimales que son infinitos, se puede hallar un límite en el infinito.

Categoría C: la mística, el alma, la eternidad, ... Dios es infinito, el amor de dios es infinito.

Categoría D: el universo, los planetas, las galaxias, ... El espacio es infinito, contar las estrellas es infinito, el cosmos está lleno de infinitas estrellas, las estrellas son infinitas en la vía láctea.

Categoría E: las ciencias naturales, ... Las especies vivientes son infinitas y aún no descubiertas, un estudio científico sobre la existencia humana es infinito, contar las arenas del mar es infinito, los cristales de la arena son infinitos. 
Dos de los docentes también citaron la frase "Hasta el infinito y más allá".

Pregunta 4. ¿Son iguales o diferentes estas tres palabras: indefinido, ilimitado, infinito? cinco docentes consideran que son palabras iguales o son sinónimos, dos docentes consideran que son palabras distintas. También se evidencia una postura potencial hacia el infinito (véase figura 18).

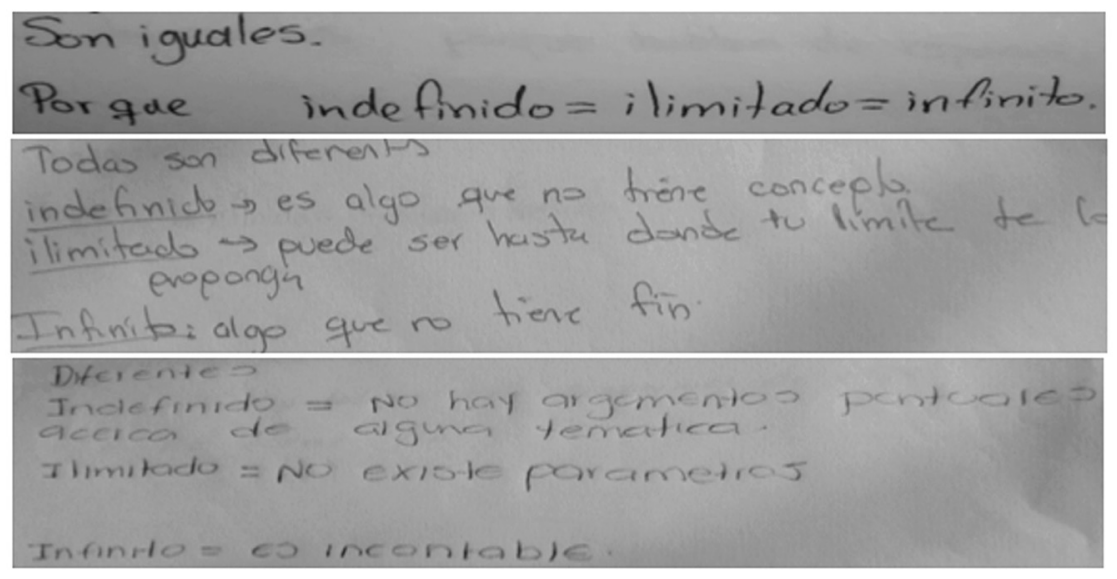

Figura 18. Evidencia de algunas respuestas de los docentes a la pregunta 4 del anexo A. Fuente: Elaboración propia

Las anteriores intuiciones están muy lejos del concepto de infinito, la etapa 3 de esta investigación evidencia la presencia de distintos obstáculos didácticos como el de la formación docente, pues, en algunas universidades con programas en educación matemática, no se han creado cátedras que permitan que futuros docentes en matemáticas reflexionen acerca del concepto de infinito en acto.

\section{Conclusiones}

El análisis del infinito no es un tema explícito de enseñanza en la formación de algunos docentes de matemáticas, lo que lleva a que cada docente en su formación cree un concepto intuitivo del infinito (obstáculo didáctico) y que luego sea transmitido a sus estudiantes. Es por ello, que vemos gran semejanza entre las concepciones de docentes y estudiantes, lo que hace difícil la argumentación en cuanto a esta temática. 
Como hemos visto en las respuestas proporcionadas en los cuestionarios, el infinito potencial tiene gran aceptación entre estudiantes y docentes, estas intuiciones llegan a ser impedimentos a la hora de aprender un infinito actual, que de hecho ayudará a una enseñanza y aprendizajes más significativos de otros objetos matemáticos. Se nota también, que a pesar de una formación universitaria en matemáticas, ante este tipo de preguntas, estamos en el mismo nivel de los estudiantes.

Por tanto, es evidente la necesidad de propuestas didácticas que ayuden a la comprensión del infinito en la formación de próximos docentes. También se requiere que la enseñanza del concepto de infinito se haga de manera explícita, para lo cual se debe incluir en el currículum escolar. De este modo cambiaríamos nociones erróneas acerca del infinito y afrontaríamos más situaciones referentes al infinito actual, dentro del aula de clase.

\section{Referencias}

Arrigo, G. y D’Amore, B. (1999). Lo veo, pero no lo creo: Obstáculos epistemológicos y didácticos en el proceso de comprensión de un teorema de Georg Cantor que involucra al infinito actual. Educación matemática, $11(1), 5-24$.

Arrigo, G., D'Amore, B. y Sbaragli, S. (2011). Infiniti infiniti. Trento: Erickson. [Versión en idioma español: (2011). Infinitos infinitos. Bogotá: Magisterio].

D’Amore, B., Arrigo, G., Bonilla Estévez, M., Fandifio Pinilla, M.I., Piatti, A., Rodríguez Bejarano, J., Rojas Garzón, P.J., Romero Cruz, J.H. y Sbaragli S. (2006). El "sentido del infinito". Epsilon, 22(2), 65, 187-216.

Dubinsky, E., K. Weller, M. McDonald y A. Brown (2005a). Some historical issues and paradoxes regarding the concept of infinity: An Apos-based analysis: Part 1, Educational Studies in Mathematics, 58, 335-359.

Fiorentini, D y Lorenzato, S. (2010). Investigación en educación matemática. Campinas, Brasil: Autores asociados.

Garbin, S. y Azcárate, C. (2001). El concepto de infinito actual. Una investigación acerca de las incoherencias que se evidencian en alumnos de bachillerato. Suma, 38, 53-67.

Garbín, S. (2005a). ¿Cómo piensan los alumnos entre 16 y 20 años el infinito? La influencia de los modelos, las representaciones y los lenguajes matemáticos. Revista Latinoamericana de Investigación en Matemática Educativa, 8(2), 169-193.

Hernández Sampieri, R., Fernández Collado, C., \& Baptista Lucio, P. (2010). Metodología de la investigación. México, DF: McGraw Hill. 
Jato Canales, S. (2012). El infinito en las Matemáticas de la Enseñanza Secundaria.

(Tesis de Maestría). Universidad de Cantabria, España.

Núñez Errázuriz R. (1991). A 3-dimension conceptual space of transformations for the study of the intuition of infinity in plane geometry. Proceedings of the 15th International Conference Psychology of Mathematics Education. II Tsukuba. $121-128$.

Ortiz, J. R. (1994). El concepto de infinito. Revista de la ASOVEMAT (Asociación Matemática Venezolana), 1(2), 59-81.

Ruiz Higueras, L. (1993). Concepciones de los alumnos de secundaria sobre la noción de función: Análisis epistemológico y didáctico. Granada, Tesis Doctoral. Universidad de Granada.

Waldegg, G. (1996). Identificación de obstáculos epistemológicos en el estudio del infinito actual. Revista Mexicana de Investigación Educativa, 1(1), 107-122.

ANEXO A: Cuestionario: intuición acerca del infinito.

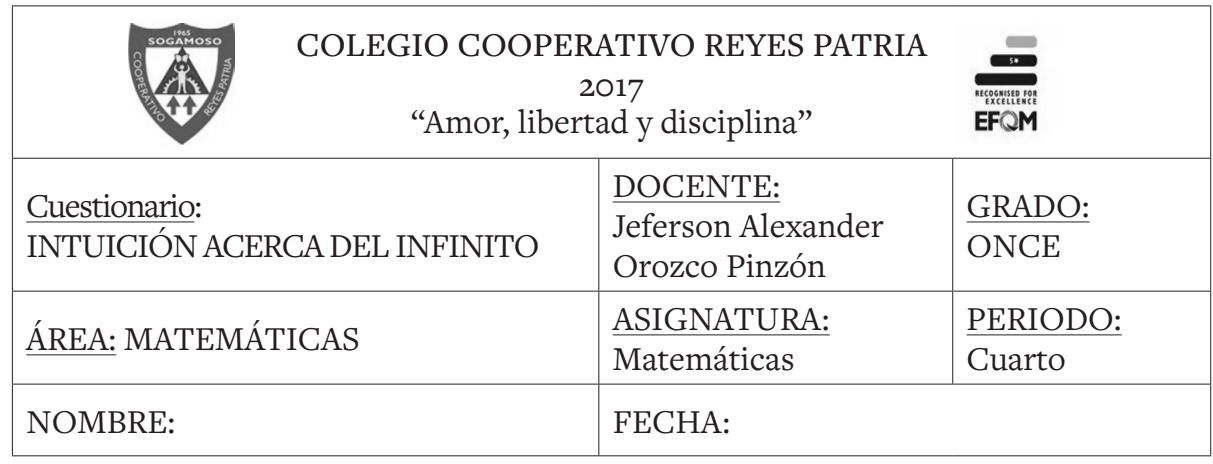

Contesta las siguientes preguntas, si lo deseas también puedes utilizar el respaldo de la hoja.

1. Desde tu percepción podrías decirme: ¿Qué es el INFINITO? y ¿Por qué es infinito?

2. Dentro de un contexto no matemático, ¿Cómo explicarías a una persona el concepto de INFINITO?

3. Escribe cinco frases (con respecto a cualquier tema, matemático o no) que contengan la palabra infinito.

4. ¿Existe alguna relación entre las palabras INDEFINIDO, ILIMITADO, INFINITO? 
ANEXO B. Cuestionario: correspondencia biunívoca.

\begin{tabular}{|l|l|l|}
\hline \multicolumn{2}{|c|}{$\begin{array}{c}\text { COLEGIO COOPERATIVO REYES PATRIA } \\
\text { "Amor, libertad y disciplina” }\end{array}$} & $\frac{\text { GRADO: }}{\text { ONCE }}$ \\
\hline $\begin{array}{l}\text { Cuestionario: } \\
\text { INTUICIÓN ACERCA DEL INFINITO }\end{array}$ & $\underline{\text { DOCENTE: }}$ \\
\hline ÁREA: MATEMÁt & $\frac{\text { PERIODO: }}{\text { Cuarto }}$ \\
\hline NOMBRE: & MSIGNATURA: & Matemáticas \\
\hline
\end{tabular}

Correspondencia Biunívoca: también llamada correspondencia uno-a-uno, se establece cuando para cada elemento del primer conjunto que se corresponde con solo un elemento del segundo conjunto, tal elemento del segundo conjunto se corresponde con solo aquel elemento del primer conjunto.

Contesta las siguientes preguntas, si lo deseas también puedes utilizar el respaldo de la hoja.

1. ¿Consideras que hay más puntos en el segmento $A B$ que en el segmento CD? ¿Puedes establecer una correspondencia biunívoca entre los puntos del segmento $\mathrm{AB}$ y el segmento $\mathrm{CD}$ ?

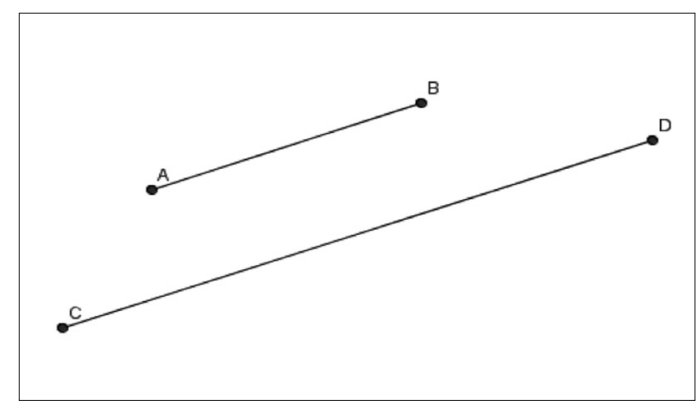

2. ¿Consideras que hay más puntos en el segmento $\mathrm{AB}$ que en la semirrecta $s$ ? ¿Puedes establecer una correspondencia biunívoca entre los puntos del segmento $\mathrm{AB}$ y la semirrecta $s$ ? 


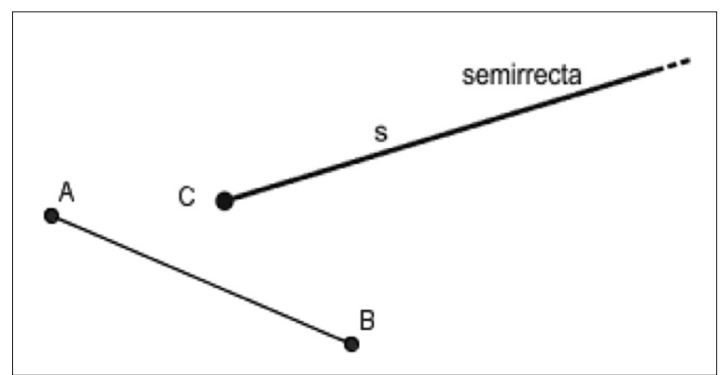

3. ¿Consideras que hay más puntos en el segmento $\mathrm{AB}$ que en la recta $r$ ? ¿Puedes establecer una correspondencia biunívoca entre los puntos del segmento AB y la recta $r$ ?

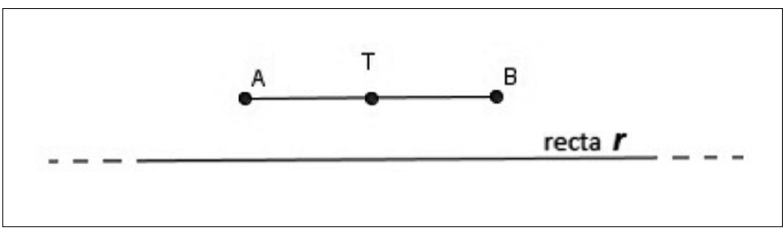

4. ¿Consideras que hay más puntos en la circunferencia de radio $R$ o en la circunferencia de radio $s$ ? ¿Puedes establecer una correspondencia biunívoca entre los puntos de la circunferencia de radio $R$ y la circunferencia de radio $s$ ?

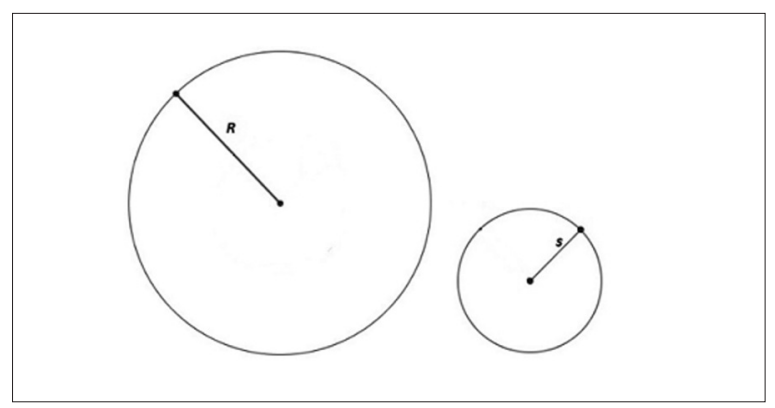

